\title{
COVID-19 Pandemic and the Debatable Use of Hydroxychloroquine
}

\section{YASHIKA KAUSHAL ${ }^{* 1}$, RATIBHA KAUSHAL ${ }^{2}$}

Healthcare professionals and scientists were not able to provide a good answer to the COVID-19 pandemic that shook the whole world. Hydroxychloroquine is an antimalarial drug with a good safety profile. It can be used in pediatric subjects as well as pregnant and breastfeeding women. Hydroxychloroquine is a widely used, essential drug in dermatology. It has got anti-inflammatory, antibacterial, antiviral and immunomodulatory properties. It was globally prescribed to prevent and treat COVID-19 caused by SARS-CoV-2 virus. The use of this drug in treating COVID-19 is debatable and for sure is not indicated in the labelling documents provided by the companies that manufacture this drug. The unnecessary use of this drug also led to short supply. We hereby review its properties, mechanism, safety profile and the issue COVID pandemic has caused to the supply of this drug.

KEYWORDS: COVID-19, Coronavirus, Hydroxychloroquine

\section{INTRODUCTION}

Hydroxychloroquine is a low-cost antimalarial derived from chloroquine with a good safety profile. It has an immunomodulatory, anti-inflammatory and photoprotective action, although it can act as a photosensitizer. In dermatology, it is indicated as first-line treatment in lupus erythematosus and is widely used off-label in multiple autoimmune/inflammatory skin diseases. ${ }^{1-3}$ It possesses antibacterial, antifungal and antiviral properties, which is why it was prescribed off-label for prophylaxis and treatment of the infection caused by the coronavirus SARS-CoV-2 (COVID-19). 4,5 The increased use of hydroxychloroquine in this setting resulted in difficulties obtaining the drug and even a temporary shortage. Below, we review its mechanism of action and toxicities, and the threat that COVID19 has posed to the supply of the drug.

Hydroxychloroquine has a high oral bioavailability; $45 \%$ is eliminated via the kidneys and it is metabolized by cytochrome $\mathrm{P} 450$, although its plasma levels are not affected by inducers or inhibitors of these enzymes. Its mechanism of action is complex. Its immunomodulatory action results from inhibition of antigen presentation through the major histocompatibility complex, stabilization of lysosomal membranes, a decrease in cell-mediated cytotoxicity and inhibition of multiple intracellular Toll-like receptors. ${ }^{3}$ Its anti-inflammatory effect is secondary to the inhibition of phospholipase A2 and $\mathrm{C}$ and various cytokines and its photoprotective effect is secondary to its antioxidant and DNAstabilizing properties, and to the reduction of interleukin levels after ultraviolet irradiation. It also decreases viral, bacterial and fungal survival in lysosomes and endosomes. ${ }^{3}$

This drug has immunomodulatory action. It inhibits antigen presentation through major histocompatibility complex, leads to stabilization of lysosomal membranes, decreases cell-mediated cytotoxicity and inhibits multiple intracellular Tolllike receptors. It has antioxidant properties and protects against UV induced free radical damage, it absorbs UV radiation, binds to DNA, regulates RNA transcription, reduces interleukin levels after UV irradiation and reduces antigenic presentation in irradiated skin. It exhibits antibacterial and antiviral action by alkalinizing intracellular phagosomes and organelles, reducing the growth and survival of intracellular bacteria and viruses and enhancing the intracellular action of antibiotics. It also inhibits post-translational viral protein modification and also inhibits sialic acid production. It is also known to have antithrombotic property wherein it inhibits platelet adhesion and aggregation, increases endothelium-mediated vasodilation, and inhibits the formation of antiphospholipid antibodies.

(C) Yashika Kaushal et al. This is an open access article distributed under the terms of the Creative Commons Attribution License CC-BY-NC 4.o, which permits unrestricted use, distribution and reproduction in any medium, provided the use is not commercial and the original author(s) and source are cited. Submitted on: 19-Aug-2021; Accepted on: 18-Nov-2021 
Hydroxychloroquine also has lipid-lowering ability. It increases the number of LDL receptors and increases lipid excretion. This drug increases insulin secretion and insulin sensitivity.

Hydroxychloroquine has a good safety profile. Its discontinuation due to adverse effects is uncommon. One of the most feared toxicities is retinal, seen in $7.5 \%$ of patients, although it is extremely rare during the first 5 years of treatment. Doses greater than 5 $\mathrm{mg} / \mathrm{kg}$ actual weight/day and duration longer than 10 years of treatment are associated with an increased rate of retinopathy. ${ }^{6}$ Gastrointestinal adverse effects are relatively common and include anorexia, pyrosis, diarrhoea and abdominal distention. The most common cutaneous adverse effects are rash, hyperpigmentation and pruritus, and it may cause photosensitivity. Other rare toxicities include cardiac, muscle, and hematologic toxicities. ${ }^{7}$ Hydroxychloroquine has powerful antimicrobial activity. An antiviral effect against influenza A and B, hepatitis $\mathrm{B}$ and $\mathrm{C}$, herpes simplex, Chikungunya, Dengue, Zika and Ebola, among others, has been described. Hydroxychloroquine was considered one of the most promising drugs for the treatment of COVID-19. Its antiviral role is based on its potential ability to inhibit virus fusion with the host cell, block viral transport from endosomes to endolysosomes and reduce the cytokine storm in severe patients. ${ }^{3}$ Antimalarials interfere with glycosylation of the angiotensin-converting enzyme receptor, a receptor used by SARS-CoV-2 to enter cells, decreasing viral penetration. They also alkalinize endosomes and endocytic vesicles, altering virus endocytosis. They also reduce the release of proinflammatory cytokines by decreasing antigenic presentation and $\mathrm{CD}_{4}+\mathrm{T}$ cell activation, and reducing intracellular signaling of Toll-like receptors. ${ }^{8,9}$

Despite the lack of consistent evidence that hydroxychloroquine was effective in the treatment or prevention of COVID-19 and although it was only recommended by the Infectious Disease Society of America within RCTs, the antimalarial was used in various hospital treatment protocols and even recommended to the general population. ${ }^{4}$ The FDA warned of the potential cardiovascular effects of the drug, then authorized its use in patients hospitalized due to COVID-19, and subsequently withdrew this authorization. $^{10}$

The high demand for the drug caused difficulties in administration and shortage.4,5 Furthermore, India, one of the largest producers of generic hydroxychloroquine, temporarily prohibited the export of this drug, which affected the global supply chain. ${ }^{4}$ In an international survey of members of the Systemic Lupus International Collaborating Clinics, $55 \%$ described a shortage of hydroxychloroquine during the pandemic among patients with SLE." Other authors reported that there was anxiety and uncertainty among individuals with SLE because they could not access the medication..$^{12,13}$ Regarding the effectiveness of hydroxychloroquine in COVID19, a recent meta-analysis that included 14 studies found no significant differences in survival, reduction in symptoms at 10 days or seroconversion rate, and described a higher rate of cardiovascular and gastrointestinal adverse effects than in the control groups..$^{10}$ A recent review of the Cochrane database found no significant differences in the risk of death from COVID-19 when using hydroxychloroquine, and the authors recommend that no further RCTs should be conducted with this drug in this disease. ${ }^{14}$ A recently published RCT did not find any positive effect of hydroxychloroquine as COVID-19 prophylaxis either. ${ }^{15}$ Hydroxychloroquine is an essential drug for the management of malaria and for the treatment of patients with certain rheumatological and/or dermatological diseases, and we believe it is important to be cautious in recommending this drug off-label for other diseases (including COVID-19), in order to ensure its supply to patients who require it.

\section{CONCLUSION}

Hydroxychloroquine is indispensable in the dermatological treatment arsenal. It is indicated in first or second line in multiple inflammatory, granulomatous, photoinduced or photoaggravated skin diseases. The treatment and prevention of COVID-19 with hydroxychloroquine has yielded unsatisfactory results and has caused difficulties in the supply of the drug. Greater caution is required when recommending large-scale use in off-label diseases, thereby ensuring access to the drug for patients who require it.

\section{REFERENCES}

1. Fernandez AP. Updated recommendations on the use of hydroxychloroquine in dermatologic practice. J Am Acad Dermatol.2017;76:1176-82.

2. Chew CY, Mar A, Nikpour M, Saracino AM. Hydroxychloroquine in dermatology: New 
perspectives on an old drug. Australas J Dermatol. 2020;61:e150-7.

3. Sardana K, Sinha S, Sachdeva S. Hydroxychloroquine in dermatology and beyond: Recent update. Indian Dermatol Online J.2020;11:453-64.

4. Cox S. To dispense or not to dispense: Lessons to be learnt from ethical challenges faced by pharmacists in the COVID-19 pandemic. Dev World Bioeth. 2020. https://doi.org/10.1111/dewb.12284

5. Baral S, Rao A, Rwema JOT, Lyons C, Cevik M, Kågesten $\mathrm{AE}$,et al. Competing health risks associated with the COVID-19 pandemic and response: A scoping review. medRxiv.

6. Melles RB, Marmor MF. The risk of toxic retinopathy in patients on long-term hydroxychloroquine therapy. JAMA Ophthalmol.2014;132:1453-6o.

7. Mohammad S, Clowse MEB, Eudy AM, CriscioneSchreiber LG. Examination of hydroxychloroquine use and hemolytic anemia in G6PDH-deficient patients. Arthritis Care Res (Hoboken).2018;70:481-5. 8. Ortega-Pena M, González-Cuevas R. Familiar dermatolo-gic drugs as therapies for COVID-19. Actas Dermosifiliogr.2021;112:118---26.

9. Stojkovic-Filipovic J, Bosic M. Treatment of COVID 19-repurposing drugs commonly used in dermatology. Dermatol Ther. 2020;33:e13829.
10. Choudhuri AH, Duggal S, Ahuja B, Biswas PS. The efficacy and safety of hydroxychloroquine (HCQ) in treatment of COVIDi9 - a systematic review and meta-analysis. Indian J Med Microbiol.2021, http://dx.doi.org/10.1016/j.ijmmb.2021.03.002.50.

11. Mendel A, Bernatsky S, Askanase A, Bae S-C, Clarke AE,Costedoat-Chalumeau N, et al. Hydroxychloroquine shortages among patients with systemic lupus erythematosus during theCOVID-19 pandemic: Experience of the Systemic Lupus Interna-tional Collaborating Clinics. Ann Rheum Dis. 2021;80:1-2.

12. Shah M, Sachdeva M, Dodiuk-Gad RP. The impact of hydroxychloroquine shortages on patients with dermatological conditions during COVID-19 pandemic. Dermatol Ther. 2020;33:e13524.

13. Peschken CA. Possible consequences of a shortage of hydroxychloroquine for patients with systemic lupus erythematosus amid the COVID-19 pandemic. J Rheumatol. 2020;47:787-90.

14. Singh B, Ryan H, Kredo T, Chaplin M, Fletcher T. Chloroquine orhydroxychloroquine for prevention and treatment of COVID-19.Cochrane Database Syst Rev. 2021;2:CDo13587.

15. Mitjà O, Corbacho-Monné M, Ubals M, Alemany A, Suner C, Tebé $C$ et al. A cluster-randomized trial of hydroxychloroquine for prevention of Covid-19. N Engl J Med. 2021;384:417-27.10 